\title{
Sex ratio of mirid populations shifts in response to hostplant co-infestation or altered cytokinin signaling ${ }^{\mathrm{FA}}$
}

Nora Adam ${ }^{1,2}$, Theresa Erler ${ }^{1}$, Mario Kallenbach ${ }^{1 \dagger}$, Martin Kaltenpoth ${ }^{3 \dagger}$, Grit Kunert ${ }^{4}$, Ian T. Baldwin ${ }^{1}$ and Meredith C. Schuman ${ }^{1,2 *}$

U 1. Department of Molecular Ecology, Max Planck Institute for Chemical Ecology, Hans-Knöll-Straße 8, 07745 Jena, Germany

2. German Centre for Integrative Biodiversity Research (iDiv), Deutscher Platz 5e, 04103 Leipzig, Germany

3. Max Planck Research Group Insect Symbiosis, Max Planck Institute for Chemical Ecology, Hans-Knöll-Straße 8, 07745 Jena, Germany

4. Department of Biochemistry, Max Planck Institute for Chemical Ecology, Hans-Knöll-Straße 8, 07745 Jena, Germany

${ }^{\dagger}$ Current address: Shimadzu Germany Ltd., Technical Office Jena, Tatzendpromenade 2, 07745 Jena, Germany

Current address: Department for Evolutionary Ecology, Johannes Gutenberg-Universität Mainz, Johann-Joachim-Becher-Weg 13, 55128 Mainz, Germany

*Correspondence: Meredith C. Schuman (mschuman@ice.mpg.de)

doi: 10.1111/jipb.12507

Abstract Herbivore species sharing a host plant often compete. In this study, we show that host plant-mediated interaction between two insect herbivores - a generalist and a specialist - results in a sex ratio shift of the specialist's offspring. We studied demographic parameters of the specialist Tupiocoris notatus (Hemiptera: Miridae) when co-infesting the host plant Nicotiana attenuata (Solanaceae) with the generalist leafhopper Empoasca sp. (Hemiptera: Cicadellidae). We show that the usually female-biased sex ratio of $T$. notatus shifts toward a higher male proportion in the offspring on plants coinfested by Empoasca sp. This sex ratio change did not occur after oviposition, nor is it due differential mortality of female and male nymphs. Based on pyrosequencing and
PCR of bacterial 16S rRNA amplicons, we concluded that sex ratio shifts were unlikely to be due to infection with Wolbachia or other known sex ratio-distorting endosymbionts. Finally, we used transgenic lines of $\mathrm{N}$. attenuata to evaluate if the sex ratio shift could be mediated by changes in general or specialized host plant metabolites. We found that the sex ratio shift occurred on plants deficient in two cytokinin receptors (irCHK2/3). Thus, cytokinin-regulated traits can alter the offspring sex ratio of the specialist $T$. notatus.

Edited by: Yonggen Lou, Zhejiang University, China Received Aug. 13, 2016; Accepted Nov. 8, 2016; Online on Nov. 14, 2016

FA: Free Access, paid by JIPB

\section{INTRODUCTION}

A battle is more complicated when fought on multiple fronts, and plant-herbivore interactions are complicated by the fact that plants often interact with multiple herbivores (Strauss 1991; Thompson 1998; Mathur et al. 2013; Stephens et al. 2013). Herbivores attacking the same host plant often compete and may affect each other's performance (Denno et al. 1995, 2000). Competition between herbivore species can influence their feeding, oviposition behaviors, spatial distribution, colonization processes and other life-history parameters (Birch et al. 1980; Cédola et al. 2013; Fragoso et al. 2014) and consequently alter population dynamics in the community (Denno et al. 1995). Changes in host plant chemistry and nutritional value can mediate these competition effects. Such changes are known to affect a variety of parameters related to herbivores' life history, including reproductive strategies (Faeth 1986; Inbar et al. 1995; Denno et al. 2000; Lynch et al. 2006). For instance, the sex ratio of herbivore populations might change in response to host plant quality and competition for limiting resources (Craig et al. 1992; Awmack and Leather 2002; Visser et al. 2014).

Sex ratio, usually expressed as the ratio of males to females in a given population, has profound effects on 
population dynamics. Fisher (1930) suggested that the evolutionarily stable strategy (ESS) of most species is to invest equally in females and males. Later, sex ratio theories predicted that shifts towards one of the sexes can occur in response to various factors (Hamilton 1967; Trivers and Willard 1973; Charnov et al. 1981; Fox et al. 1990; West and Sheldon 2002). Among these are the local resource enhancement (LRE) and local resource competition (LRC) theories. The LRE predicts that the sex ratio will be shifted towards one sex when producing this sex results in a higher fitness return for the parents. The LRC predicts that if competition among individuals of a particular sex is higher, then the sex ratio will be biased towards the less competing sex (Hamilton 1967; West 2013). Environmental conditions might also play a role in biasing sex ratios of different populations (Shine 1999; West et al. 2002). However, biased sex ratios might simply resulted from differential mortality of female and male juveniles. Furthermore, endosymbionts, which manipulate the reproductive systems of their hosts can cause female-biased sex ratios in insects, the most commonly known being Wolbachia (Werren 1997; Werren and Beukeboom 1998).

An example of plant-mediated herbivore competition is the interaction between the generalist Empoasca sp. (Hemiptera: Cicadellidae) and the solanaceous specialist Tupiocoris notatus (Hemiptera: Miridae) when both co-infest the host plant Nicotiana attenuata (Solanaceae). Annual N. attenuata plants germinate after fire and in disturbed areas in western North America; within populations, plants differ in their jasmonate (JA) defense hormone biosynthesis, signaling, and associated defense metabolites (Schuman et al. 2009; Kallenbach et al. 2012). Empoasca sp. leafhoppers are phloem and cell content feeders, which feed preferentially on plants with reduced JA accumulation (Kallenbach et al. 2012). T. notatus frequently colonize N. attenuata (Kessler and Baldwin 2001) regardless of JA accumulation. However, prior attack of Empoasca sp. on JA-deficient $N$. attenuata plants is correlated with reduced damage by $T$. notatus on the same plants (Fragoso et al. 2014).

We therefore hypothesized that Empoasca sp. co-infestation of $\mathrm{N}$. attenuata plants affects other life history parameters of $T$. notatus such as numbers of progeny and their sex ratio, since this might explain avoidance of Empoasca sp. by T. notatus. We report a shift in the female-biased sex ratio of T. notatus towards males when Empoasca sp. co-infested plants. We further investigated the sex ratio shift by addressing three hypotheses. First, we hypothesized that the sex ratio shift resulted from differential mortality of female and male nymphs; second, that it was due to altered infection with endosymbionts that are known to manipulate the reproduction of their host; and third, that it was due to changes in host quality. To test the third hypothesis, we used several transgenic lines that are enhanced or reduced in certain metabolites. Local increase of cytokinin (CK) levels results in increasing $T$. notatus damage on $N$. attenuata (Schäfer et al. 2013). Therefore, we used plants with increased levels of CKs and total protein (SAGipt4) as well as plants silenced in two of the three predicted CK receptors (irCHK2/3) resulting in reduced defense induction (Schäfer et al. 2015) and presumably lower levels of primary metabolites (Werner et al. 2008). In addition, we used plants with either decreased (irGGPPS) or increased (irJAZh) levels of 17-hydroxygeranyllinalool diterpene glycosides (DTGs): specialized metabolites known to also affect $T$. notatus damage levels and other herbivores in the natural community of N. attenuata (Heiling et al. 2010; Oh et al. 2012) and finally, plants with reduced photosynthetic rate and increased levels of RuBP protein (irRCA; Mitra and Baldwin 2008, 2014). Since many sex ratio theories predict a role of resources or food in sex ratio allocation, our last hypothesis aims to target a specific host plant pathway involved in regulating sex ratio shifts in the specialist $T$. notatus.

\section{RESULTS}

Empoasca sp. co-infestation shifts the female-biased sex ratio of Tupiocoris notatus towards males

We first investigated the consequences for $T$. notatus when Empoasca sp. co-infests its host plant. Since Empoasca sp. attack only naturally or experimentallyproduced jasmonate (JA)-deficient genotypes of $N$. attenuata (Kessler et al. 2004; Kallenbach et al. 2012), we used an inbred line rendered deficient in JA by silencing the biosynthetic gene LIPOXYGENASE 3 using RNAi, antisense (as) LOX 3 . Using asLOX3 rather than naturally JA-deficient wild genotypes allowed us 
to use the same single genotype of $\mathrm{N}$. attenuata for all experiments, avoiding confounding effects due to plant genotype variation.

Each asLOX3 plant was either infested only with T. notatus, or co-infested with Empoasca sp. and T. notatus (Figure $1 \mathrm{~A}$ ). The total number of $T$. notatus progeny did not differ between treatments (quasipoisson $G L M, d f=14, t=-0.667, P=0.516$; Figure $1 B$ ), nor did total numbers of females and males (quasipoisson GLM, $d f=14, \quad t=-1.631, \quad P=0.127$ and $t=0.255$, $P=0.803$, respectively; Figure $S 1)$. Furthermore, neither the number of eggs laid by T. notatus (quasipoisson GLM, $d f=29, t=0.048, P=0.962$; Figure $1 C)$ nor the survival of $T$. notatus adults (Cox proportional hazard model, $Z=-0.751, P=0.453$ ) were affected by Empoasca sp. co-infestation. In addition, the survival among females and males did not differ (Cox proportional hazard model,
$Z=-1.525, \quad P=0.127 ;$ Figure S2). Finally, $T$. notatus survival was also not affected by the interaction between the treatment (co-infestation) and the sex type (Cox proportional hazard model, $Z=0.819, \quad P=0.413$; Figure $\left.S_{2}\right)$. The mean survival $( \pm S E)$ for females and males in the control treatment (- Emp.) was $19.64 \pm 1.11$ days and $22.88 \pm 1.42$ days, while it was $18.70 \pm 1.32$ days and $19.85 \pm 1.68$ days in the treatment with Empoasca sp. (+Emp.).

Naturally occurring populations of $T$. notatus infesting two native populations of $\mathrm{N}$. attenuata also showed female-skewed sex ratios, although the degree of bias differed ( 0.39 vs 0.83 male/female, Figure 2 ). Further, the female-biased sex ratio of $T$. notatus in the glasshouse colony (0.86) was shifted toward males in progeny with Empoasca sp. co-infestation (sex ratio $=1.13$, binomial GLM, $d f=14, Z=3.521,{ }^{* * *} P<0.001$;
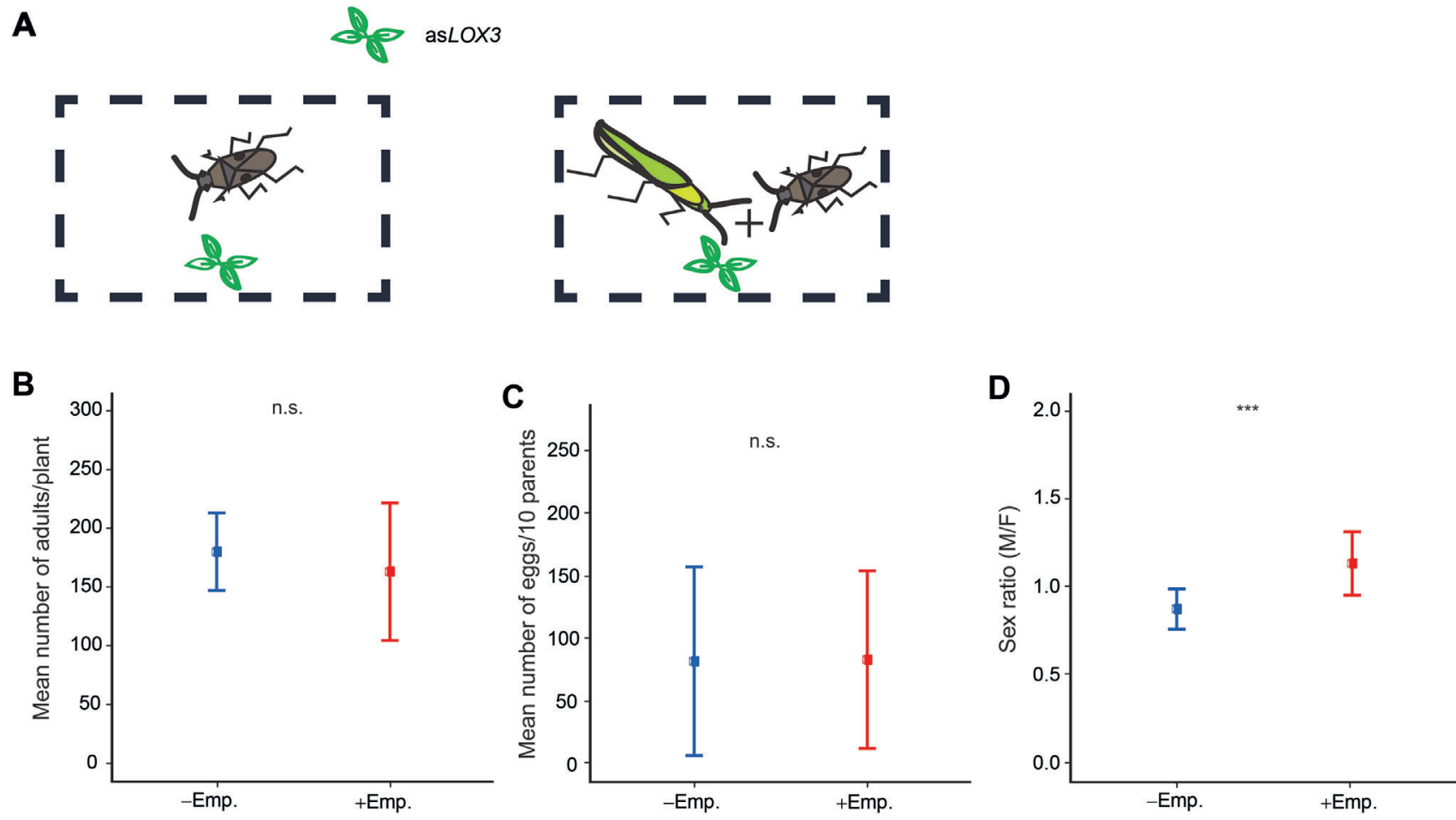

Figure 1. Empoasca sp. co-infestation of host plants shifts the sex ratio of Tupiocoris notatus progeny towards males (A) Nicotiana attenuata plants rendered jasmonate-deficient using an antisense LOX 3 construct (asLOX3) were infested either with T. notatus or simultaneously with Empoasca sp. and T. notatus. (B) The mean number of T. notatus adults did not differ between treatments. (C) In an additional setup, when T. notatus were allowed to oviposit on asLOX 3 N. attenuata plants that either simultaneously infested with T. notatus and Empoasca sp. or only with T. notatus for a total period of 3 weeks, the number of eggs laid did not differ between treatments ( $n=5$ plants and parents were moved to new plants every week for a period of three weeks). (D) Under standard experimental conditions, the sex ratio (male/female) of T. notatus is female-biased. However, in the presence of Empoasca sp. the sex ratio shifts toward males. n.s. = not significant, ${ }^{* *} P<0.001 ; G L M ; n=7$ plants in the control condition, and $n=8$ in the treatment with Empoasca sp., 10 (six females + four males of T. notatus) insects/species/plant. Graphs show mean \pm SD. 
A

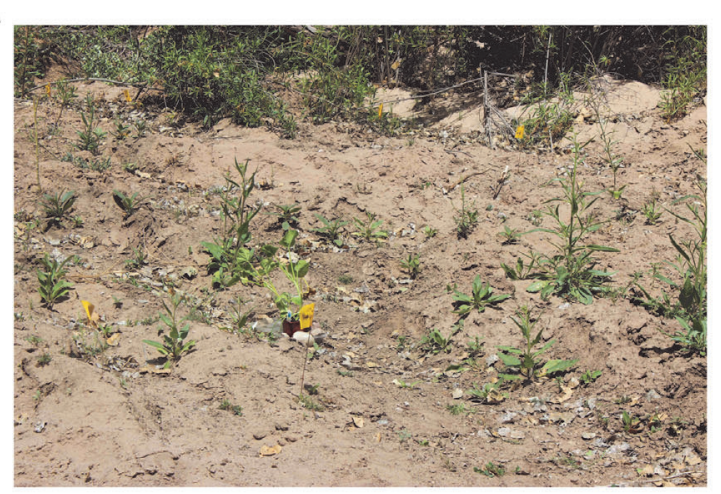

B

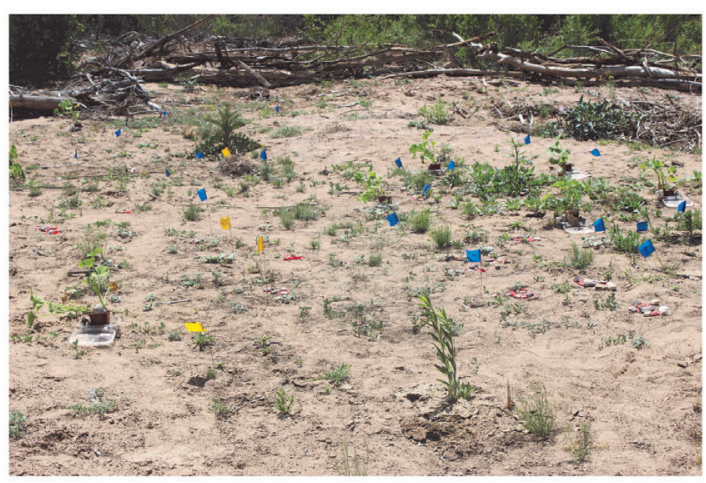

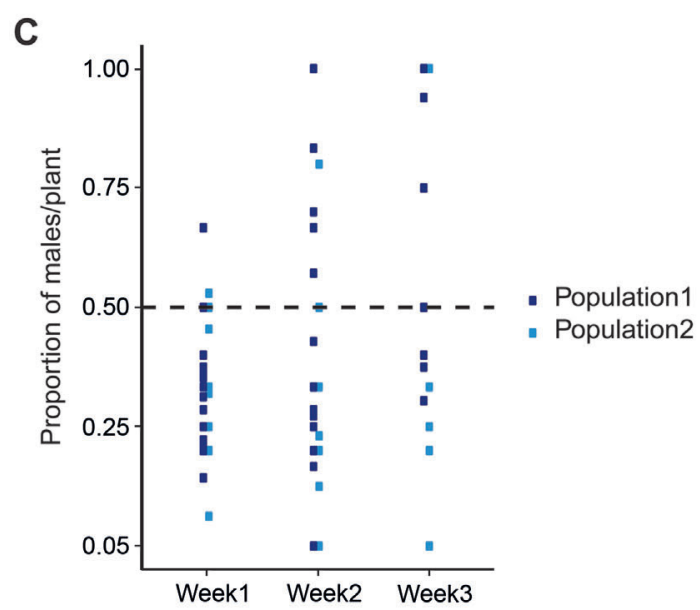

Figure $1 D)$. In order to determine whether the JA pathway is involved in the sex ratio shift, the female and male number as well as the sex ratio of $T$. notatus was recorded on JA-deficient plants (asLOX 3 ) and on control (WT) plants. The female and male number did not differ between asLOX3 and WT control plants (quasipoisson $G L M, d f=19, t=0.558, P=0.584, t=0.391, P=0.700$, respectively; Figure $3 \mathrm{~A}$ ), nor did the sex ratio (0.80 vs. 0.79 , quasibinomial $G L M, d f=19, t=-0.267, P=0.792$; Figure 3B).

\section{Empoasca sp. co-infestation affects the sex ratio of T. notatus before oviposition}

The number of $T$. notatus adult progeny (quasipoisson $\mathrm{GLM}, d f=8, t=0.458, P=0.661$; Figure $4 \mathrm{~B}$ ) and their sex ratio (binomial $G L M, d f=8, Z=-0.79, P=0.428$; Figure $4 \mathrm{C}$ ) were independent of Empoasca sp. coinfestation when Empoasca sp. were added after T. notatus parents had first been given one week to mate and oviposit on asLOX 3 plants. T. notatus parents had been removed to ensure that all oviposition occurred prior to Empoasca sp. addition. Similarly,
Figure 2. The sex ratio of $T$. notatus under natural conditions

T. notatus has a female-biased sex ratio in natural populations of N. attenuata plants. Graphs show the proportion of males on each surveyed plant. (A, B) T. notatus female and male adults were surveyed once per week for three weeks in two natural populations of $N$. attenuata $(n=16$ plants in population 1 and $n=10$ plants in population 2). (C) The average sex ratio (male/female) per population over the three weeks was 0.83 for population 1 and 0.39 for population 2 .

when $1^{\text {st }}$ instar nymphs of T. notatus were either caged alone or together with Empoasca sp. on asLOX3 plants, Empoasca sp. co-infestation did not affect the number of nymphs that reach adulthood (poisson GLM, $d f=9, Z=-0.465, P=0.642$; Figure $4 E$ ) or their sex ratio (binomial $G L M, d f=9, Z=0.842, P=0.400$; Figure $4 \mathrm{~F}$ ). This suggests that the sex ratio shift which is observed in $T$. notatus under co-infestation with Empoasca sp. occurs prior to oviposition by T. notatus mothers.

\section{Wolbachia and other endosymbionts known to} manipulate the reproductive system of their host do not account for the sex ratio shift

$T$. notatus females from the two native populations shown in Figure $2 \mathrm{~A}$ and $\mathrm{B}$, and from the glasshouse colony in Isserstedt, as well as an Empoasca sp. sample from the Isserstedt colony, were screened for Wolbachia infection by PCR according to Braig et al. (1998). The sex ratio of $T$. notatus progeny on WT plants, which is female-biased (Figure 3B) reflects the sex ratio in the Isserstedt colony. The insects in our colony are also 

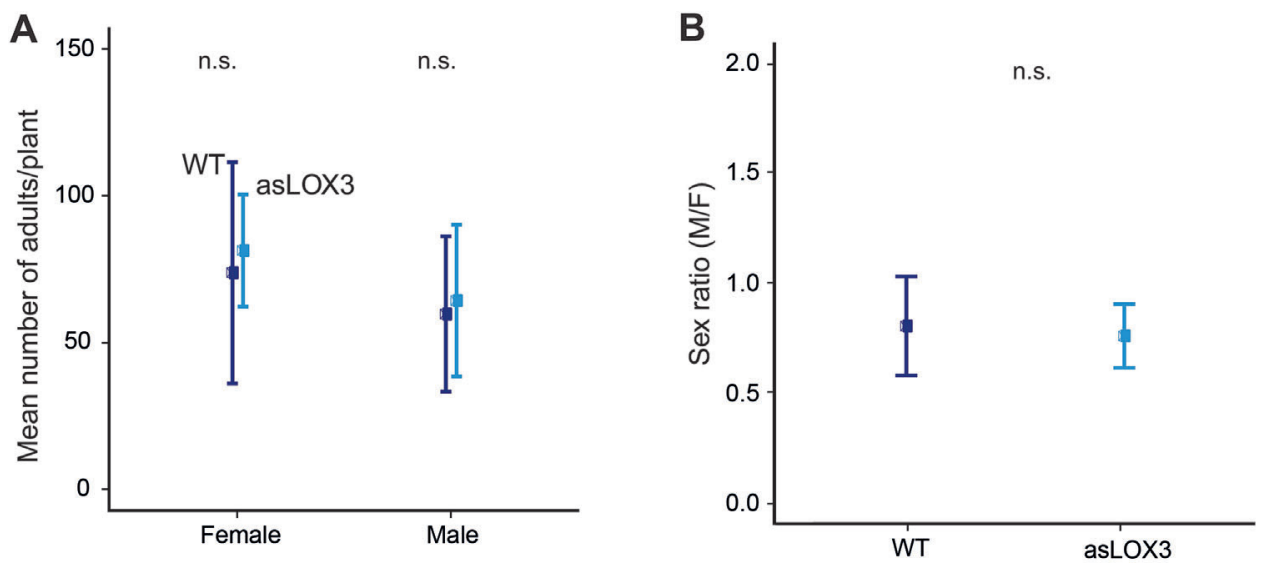

Figure 3. The number of T. notatus progeny (females and males) and their sex ratio on WT and asLOX 3 plants Neither the number of female and male of T. notatus adult progeny nor their sex ratio differed on WT versus asLOX 3 plants. Graphs show mean \pm SD. WT and asLOX 3 N. attenuata plants were infested with T. notatus. (A) The number of female and male adult progeny of T. notatus and (B) the sex ratio did not differ between genotypes. GLM; $n=10$ plants, 10 (six females + four males) insects/plant.

reared on WT plants. Only one T. notatus sample (N6), collected from a native population, tested positive for Wolbachia (Figure S3).

These data were consistent with results from pyrosequencing of the $16 \mathrm{~S}$ rRNA sequence extracted from whole T. notatus ( $n=9$ females) and Empoasca sp. ( $n=1$ pool of five adults). In total, 127,697 reads were obtained, 84,212 of which passed quality filtering (mean \pm standard error $=8,421 \pm 2,088$ per sample). Rarefaction analyses indicate that the microbiota associated with the individual insects was exhaustively sampled in samples N2, N6, N7, N8, and N9 (Figure 5). Based on $97 \%$ similarity clustering with cdhit and uclust, the sequences were grouped into 828 OTUs (see OTU tables $S_{1}$ and $S_{2}$ and representative sequence set in file S1; sequence data are available in GenBank under BioProject accession number PRJNA338226). The glasshouse-reared individuals showed generally low diversity, due to the high abundance of a single OTU closely related to Serratia marcescens, which may represent a pathogen infection in the culture. This OTU was virtually absent from the field-collected individuals, which exhibited much higher diversity. Wolbachia was present in only one sample (N6), where it represented the major OTU. No other known reproductive manipulators could be detected (i.e., Cardinium, Arsenophonus, Spiroplasma, Rickettsia). The Empoasca sp. sample yielded a very low number of sequences, which prevented solid conclusions on the microbiota composition in this sample. However, no conspicuous taxon was detected that is likely to represent an obligate mutualist.

\section{Host plant cytokinin (CK) signaling affected the sex ratio and number of female $T$. notatus progeny, likely by altering the nutritional quality of the host}

In order to determine whether the change in sex ratio of T. notatus offspring caused by co-infestation of host plants with Empoasca sp. might be due in part to changes in host quality, T. notatus parents were reared on wild-type control plants (WT) of the genotype used to generate the asLOX 3 transformed line, as well as on other transformed lines in which senescence was suppressed by increasing the levels of CKs (SAGipt4), plants silenced in two of the three predicted CK receptors (Schäfer et al. 2015) resulting in reduced defense induction and lower levels of general metabolites (irCHK2/3), and plants with decreased levels of 17-hydroxygeranyllinalool diterpene glycosides, DTGs (irGGPPS), which have been shown to receive more damage from T. notatus in nature (Heiling et al. 2010). In contrast, CKs increase T. notatus damage (Schäfer et al. 2013). The sex ratio of $T$. notatus on $\operatorname{irCHK2/3}(0.96)$ differed significantly from WT control plants (0.80) (binomial $G L M, d f=10, Z=2.087, P=0.037$; Figure $6 \mathrm{~A}$ ). The total number (females and males) of adult progeny of T. notatus tended to be lower on $\operatorname{irCHK} 2 / 3$ plants (quasipoisson GLM, $d f=10, t=-2.188, P=0.056$ ). This could be attributed to the decreased number of 


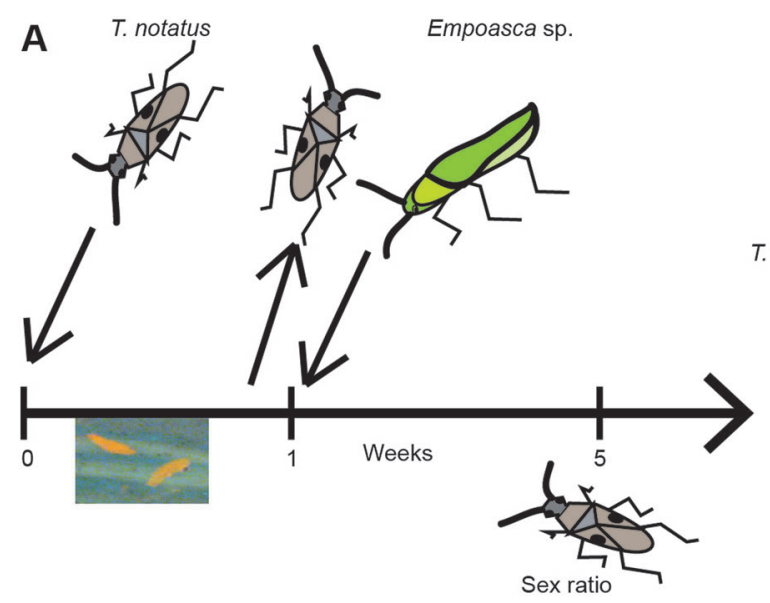

D
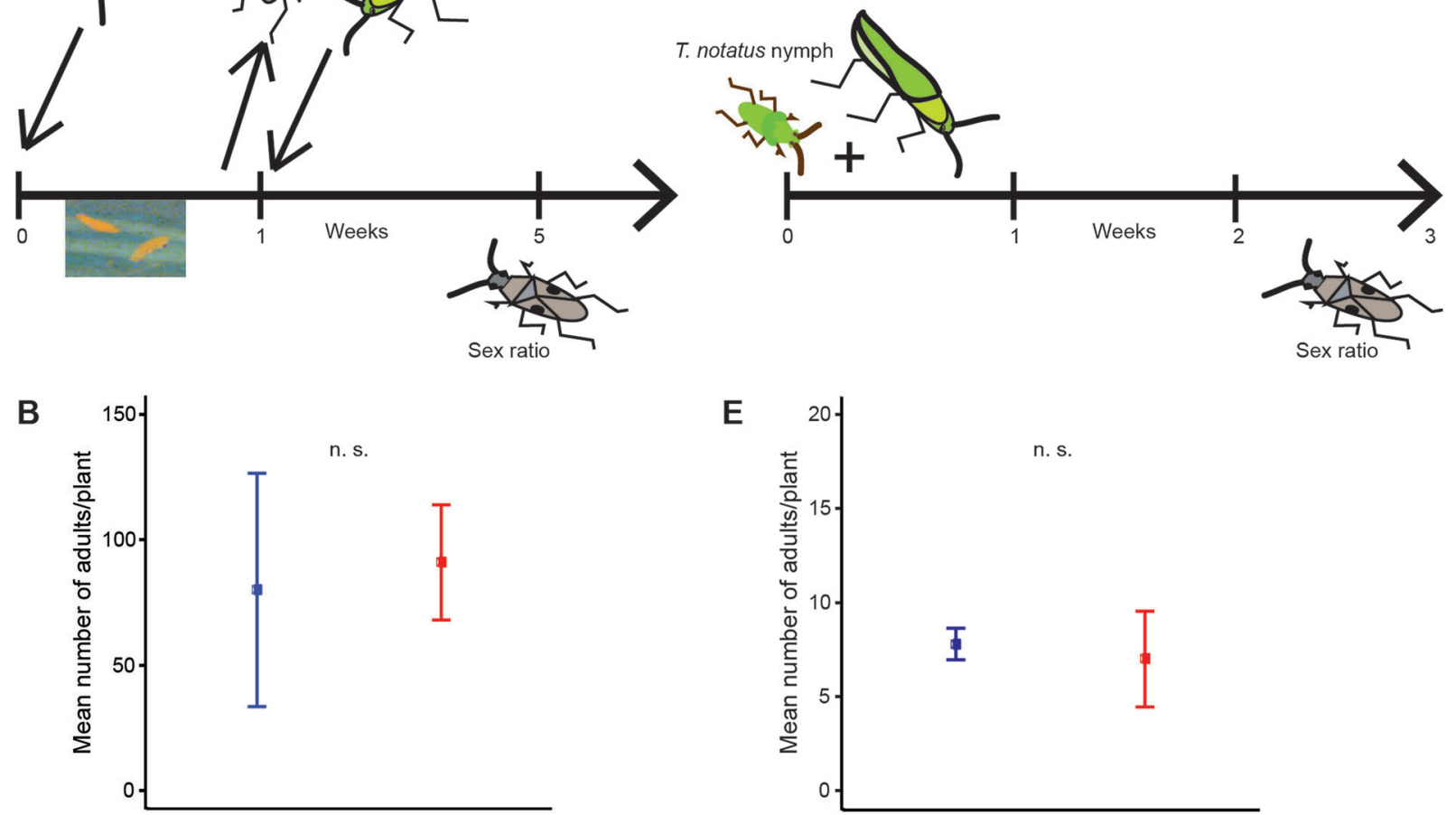

C

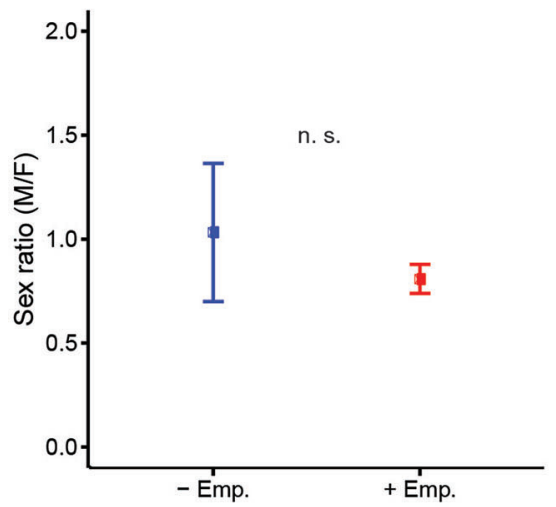

E

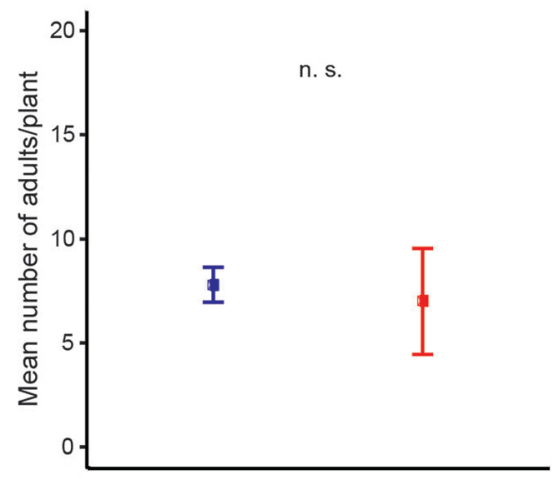

$\mathbf{F}$

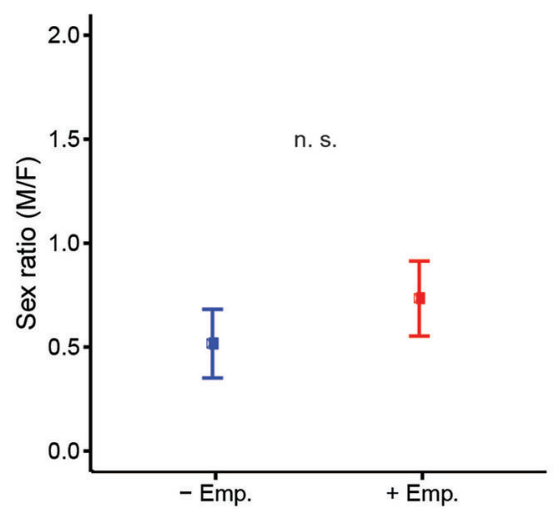

Figure 4. Empoasca sp. co-infestation of host plants does not alter the sex ratio of $T$. notatus once oviposition by T. notatus has already occurred

T. notatus parents were removed from asLOX3 plants after one week of oviposition and Empoasca sp. were added to five plants. (A) Hatched nymphs from control (uninfested) and Empoasca sp. co-infested plants were monitored until adulthood and the number and sex of those adults were determined. (B) The total number of emerged adults from eggs on plants infested with Empoasca sp. did not differ significantly from the control. (C) Additionally, the sex ratio did not differ significantly between control and Empoasca sp. co-infested plants, although the range was narrower on Empoasca sp.-attacked plants. n.s. $=$ no significant difference, GLM; $n=5$ in +Emp. and $n=4$ plant in -Emp., 10 (six females + four males of T. notatus) insects/species/plant. First-instar nymphs of T. notatus were caged either alone (control) or with adults of Empoasca sp. ( $n=5$ plants, 10 Empoasca sp. per asLOX 3 plant). (D) Nymphs from both treatments (control and Empoasca sp. co-infested plants) were monitored until adulthood and their number and sex were determined. (E, F) The number of nymphs reaching adulthood as well as their sex ratio did not differ between control and co-infested plant. n.s. = no significant difference, GLM; $n=5$ plants, 10 insects/species/plant. Graphs show mean \pm SD. 


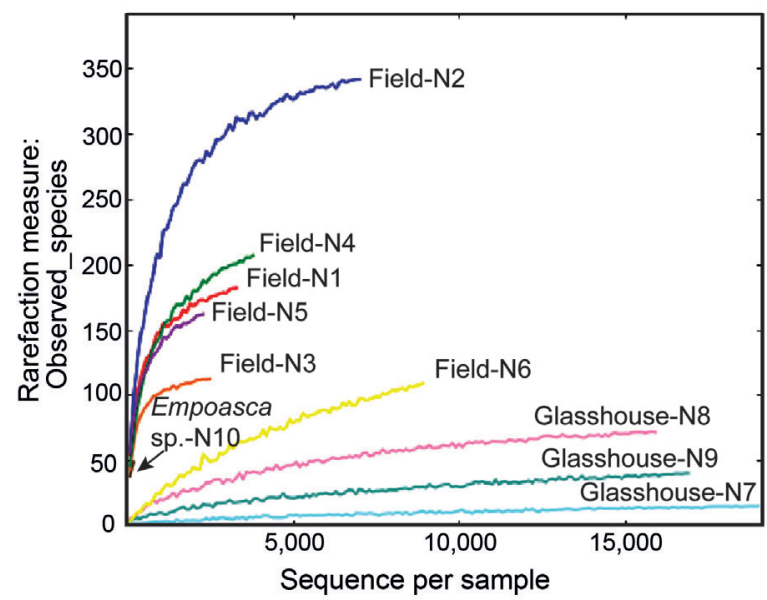

Figure 5. Rarefaction analysis of bacterial communities associated with $T$. notatus and Empoasca sp. samples Samples are $T$. notatus females collected from the field (N1-N6), from the glasshouse colony after the insects fed on asLOX 3 plants for one week (N7-N9), and a pooled sample of Empoasca sp. individuals (five insects) from the glasshouse colony (N10).

female progeny on irCHK2/3 (quasipoisson GLM, $d f=10$, $t=-2.784, P=0.021$; Figure $6 \mathrm{~B}$ ); the male number did not differ (quasipoisson GLM, $d f=10, t=1.313$, $P=0.222$; Figure $6 \mathrm{~B}$ ). In contrast, the numbers of female and male adult progeny, and the sex ratio, did not differ significantly between any other tested lines and WT plants $(P>0.4$; Figures $6 C, D, S 4 A, B)$.

In addition, one to two plants each of a line with higher levels of DTGs and other chemical resistance traits (irJAZh; $n=1$ ) (Oh et al. 2012), and a line with reduced photosynthetic rate and increased protein levels due to an increase in RuBP (irRCA, $n=2$ ) (Mitra et al. 2008, 2014) were screened. We included these lines to provide perspective on the results with lines having higher replication. The sex ratio on these plants was 1.05 and 0.99 (Figure S4B), respectively. This is consistent with the hypothesis that changing the nutritional quality of the host plant may increase the male:female ratio of $T$. notatus progeny.

\section{DISCUSSION}

Plant traits shape herbivore community composition and can determine the likelihood that two herbivore species compete over the same host plant. To understand the interactions of multiple herbivores sharing the same host plant requires assessing lifehistory parameters of these herbivores in order to determine which aspects are most affected. In this study, we show that the natural female-biased sex ratio of the solanaceous specialist $T$. notatus on the host plant N. attenuata shifted toward a higher proportion of male progeny produced on plants co-infested with the generalist leafhopper Empoasca sp. While the sex ratio of T. notatus was affected by co-infestation with Empoasca sp., other life history parameters of T. notatus, such as parental survivorship, numbers of eggs and progeny (females and males), were not significantly affected.

Sex ratio is an important demographic parameter for understanding population dynamics. Many theories including the local resource enhancement or competition (LRE and LRC) predicted a deviation from an equal investment in female and male progeny, leading to biased sex ratios in different species. In general, a decrease in the proportion of females in a population might increase local mate competition among males. Fewer females in a population could decrease population sizes in future generations, because females are often more limiting than males for the number of offspring produced; and potentially, in extreme situations, a reduction in females can cause population extinction (Hamilton 1967; Myers 1978). To determine whether this would be the case for T. notatus, the growth rate of $T$. notatus populations over multiple generations would need to be tested under differing sex ratios. It is unknown whether a male-biased sex ratio would persist in further generations of $T$. notatus.

We calculated the projected increase in $T$. notatus population in future generations based on the usually female versus more male-biased sex ratio and the mean number of adult progeny, as well as the mean female number produced on plants co-infested with Empoasca sp. We estimated that the population of $T$. notatus would drop by almost $44 \%$ after three generations. Here, we assumed that the male-biased sex ratio and the number of adult progeny per female were constant over multiple generations and ignored all external mortality factors. To accurately predict the consequences of the sex ratio shift, a modeling approach based on additional T. notatus life-history traits as well as population dynamics under natural conditions would be required. However, under natural conditions, T. notatus adults might rather disperse to Empoasca 

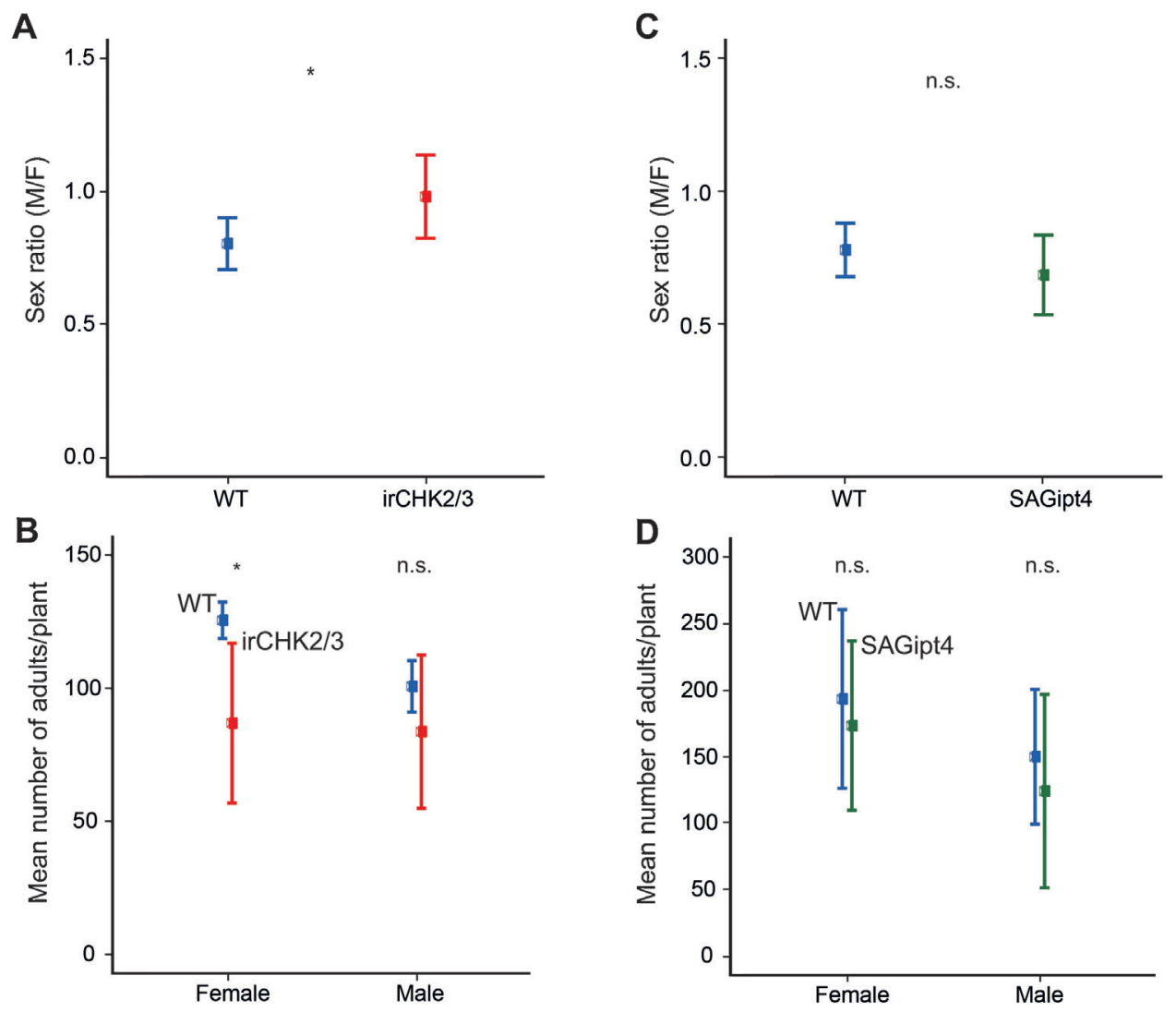

Figure 6. Alteration of plant cytokinin (CK) signaling also shifted the sex ratio of T. notatus progeny Early elongating N. attenuata plants silenced in two CK receptors (irCHK2/3) and wild-type plants (WT) were infested with T. notatus. In addition, flowering N. attenuata plants with increased levels of CKs (SAGipt4) and flowering WT plants were also infested with $T$. notatus. The numbers of female and male adult progeny as well as the sex ratio were recorded on these plants. (A) The sex ratio of T. notatus on irCHK2/3 was shifted towards an equal ratio in comparison to the natural female-biased sex ratio of T. notatus as shown on WT plants. (B) The number of female, but not of male progeny also significantly differed between irCHK2/3 and WT plants. ${ }^{*} P \leq 0.05, G L M ; n=6$ for control plants, and $n=5$ for $\operatorname{irCHK} 2 / 3,10$ (six females + four males) insects/plant. (C, D) The sex ratio and the number of female and male adult progeny between flowering SAGipt4 and control plants did not differ. GLM; $n=5$. 10 (six females + four males) insects/plant. Graphs show mean \pm SD, n.s. $=$ no significant difference.

sp.-free plants to avoid the negative impact of the interspecific interaction. Empoasca sp. attacks mainly jasmonate-deficient (JA-deficient) plants (Kallenbach et al. 2012), and therefore an increase in the frequency of JA-deficient plants may result in a higher attack rate by Empoasca sp. Under such conditions, a less femalebiased sex ratio of $T$. notatus populations is more likely to persist. Thus, it would be interesting to investigate the frequency of JA-deficient plants in native populations of $N$. attenuata in relation to the sex ratio in local T. notatus populations.

We investigated the mechanism behind the sex ratio shift of T. notatus when N. attenuata is co-infested with Empoasca sp. We demonstrated that the sex ratio shift is not due to differential female and male nymphal mortality and in fact does not occur unless parents themselves are exposed to Empoasca sp. co-infestation. The sex ratio shift is also unlikely to be due to cytoplasmic factors such as Wolbachia or to infection with other symbionts known to cause sex ratio distortion, since we rarely detected infection with any of these symbionts in multiple female-biased populations. Thus, by elimination, it seems likely that T. notatus mothers selectively produce a lower proportion of female offspring on plants co-infested with Empoasca sp.

Sex ratio theories suggest that mothers will allocate different amounts of resources when the fitness returns 
from female and male progeny differ (Charnov et al. 1981). In addition, mothers may optimize the sex ratio of their progeny to maximize benefits in the future (Mousseau and Fox 1998). Therefore, we asked whether the host plant quality as resource for the mother and the developing progeny might be involved in the sex ratio shift. Plant quality can reflect various parameters, such as the level of nutrients and defense metabolites in the plant. Many studies also reported a role of host plant quality in altering life-history traits of herbivores, including sex ratio (Awmack and Leather 2002). For example, Craig and colleagues (1992) showed that the sex ratio of sawfly populations varied in response to plant quality. When plant growth was slow, indicating lower host quality, the sex ratio was skewed towards males. The same pattern was observed in other organisms. For example, the sex ratio of the parasitoid wasp Diadegma insulare Cress. (Hymenoptera, Ichneumonidae) varied in response to the quality of their larval host (diamondback moth) and the host plant of the larvae (Fox et al. 1996).

Variation in plant quality is a broad concept and, as previously mentioned, depends on nutrients as well as defensive specialized metabolites. Jasmonate (JA) defense hormone signaling and accumulation determines which plants are subject to co-infestation by Empoasca sp. and consequently by $T$. notatus (Kessler et al. 2004; Kallenbach et al. 2012; Fragoso et al. 2014). However, the data presented here indicate that $\mathrm{JA}$ is unlikely to be involved in the sex ratio shift of $T$. notatus, since the sex ratio on JA- deficient plants (asLOX3) was similar to that on WT plants. Rather, we could narrow down plant quality aspects and demonstrate that the cytokinin (CK) pathway could influence the sex ratio of $T$. notatus at a similar rate to that observed in Empoasca sp.co-infested plants. Furthermore, T. notatus produced relatively fewer adult progeny on these plants than on wild-type (WT) control plants. The reduced total number resulted from a reduction in the female adult progeny on those plants. Interestingly, the plant parasitic cyst nematode (Heterodera schachtii) also produced fewer female progeny on Arabidopsis thaliana double knockout mutants of the two CK receptors (ahk3/4) and on transgenic lines overexpressing CK degradation enzymes (CK oxidase genes, CKXs) (Siddique et al. 2015). CKs are involved in plant growth, development, responses to biotic and environmental stress, and other important aspects of plant life history (Werner and Schmülling 2009; Ha et al. 2012). The role of CKs in delaying senescence and in nutrient mobilization to regulate source-sink relationships (Balibrea Lara et al. 2004) is probably critical for the nutritive value of the host plant. Factors such as the amount and distribution of amino acids, carbohydrates, lipids, and minerals, including the interaction among these compounds, have large effects on many life-history parameters of insects (Awmack and Leather 2002).

Based on our data, we suggest that the cost of resource investment in producing a female $T$. notatus could be greater than the cost of producing a male. If this is the case, it might be advantageous to shift to a more male-biased sex ratio of T. notatus on a host with poor quality. Indeed, the data from the experiments presented here are consistent with this idea. T. notatus progeny from parents reared on lines thought to have better nutritional value as a result of uncompromised or enhanced general metabolism (SAGipt4, WT and uninfested asLOX 3 ) tended to be more female-biased overall in comparison to less nutritious lines (irCHK2/3 and Empoasca sp. co-infested asLOX3 plants). In addition, a few replicates were included of lines altered in other aspects of host plant quality: one line having reduced photosynthetic rates (irRCA) and another with elevated jasmonate-mediated defenses (irJAZh), and both showed a shift toward higher male proportion in the progeny. These are preliminary data due to low replicate numbers of these lines, and would need to be confirmed with additional replicates in future experiments. If reproducible, these results support the conclusion that altered plant nutritional quality can explain the sex ratio shift. In general, adjusting the proportion of female progeny of $T$. notatus on a host with poor quality might also be a strategy to reduce competition, increase genetic diversity and enhance overall reproductive outcome in this sub-optimal context.

In our experiments, we used two transgenic lines targeting the CK pathway and having opposite effects on this group of plant hormones. The sex ratio on irCHK2/3 plants, which are deficient in CK signaling, was less female-biased in comparison to WT plants. SAGipt4 has increased levels of CKs and total protein and thus we might expect that the female proportion on these lines would be higher than on WT plants. However, the sex ratio remained similarly female-biased on SAGipt4 
and WT plants. This may reflect other limitations on degree of sex ratio bias. For example, increasing the female proportion in the population further might result in competition among these females for mating and oviposition sites. We propose that the sex ratio of $T$. notatus observed on WT plants in the glasshouse experiment might reflect the optimal sex ratio under these conditions and this might explain why the sex ratio did not differ between WT and SAGipt4, and in general why we never observed an increase in female bias in our glasshouse experiments. T. notatus specializes on a few host plant species and observations from over 10 years of field research in our department indicate that $T$. notatus is nearly always associated with Utah populations of $\mathrm{N}$. attenuata, so we might expect it to be well adapted to feed on our Utah WT N. attenuata plants. Although we sometimes observed a greater female bias in natural $T$. notatus populations than in our glasshouse colony ( 0.83 and 0.39, Figure 2, vs 0.8 on WT plants in glasshouse experiment, Figure $3 \mathrm{~B}$ ), the sex ratios in natural populations are likely affected by dispersal.

Empoasca sp. attack is known to downregulate many photosynthesis-related genes (Kessler et al. 2004). Further, Empoasca sp. attack might affect CKregulated nutrients, thus reducing plant quality. We propose that it is at least in part as a consequence of this change in nutritional quality that a shift in the sex ratio of $T$. notatus occurs on these plants. This hypothesized reduction of host quality caused by Empoasca sp. co-infestation is sufficient to affect the sex ratio but did not affect the total number of adult progeny. However, understanding the mechanism by which Empoasca sp. co-infestation affects $T$. notatus sex ratio requires further investigation. Sex ratio distortion is complex because a variety of mechanisms such as genic, chromosomal, cytoplasmic, or environmental principles can be involved in sex ratio determination (Robinson 1983). Thus, further experiments addressing the different aspects of sex determination mechanisms are needed to understand the mechanism behind the observed sex ratio shift. For example, identifying the chromosomal sex determination system of $T$. notatus and subsequently addressing the role of non-genetic sex-determination factors that might also shape the sex ratio would be required. The ability of $T$. notatus parents to assess plant quality and therefore adjust the sex ratio of their offspring should be better understood in order to provide a comprehensive understanding of the sex ratio shift.

N. attenuata plants are attacked by various herbivore species throughout their life cycle. Other herbivores including chewing insects might also affect life-history parameters of $T$. notatus when co-infesting $N$. attenuata plants. In particular, specialized lepidopteran chewing herbivores, larvae of Manduca sexta and $M$. quinquemaculata, remove substantial amounts of leaf area and elicit strong plant defense responses (Halitschke et al. 2001). Although examples of competition between insects from the same feeding guild are more common in literature, there are reports on interspecific competition between chewing and sucking insects (Denno et al. 1995). Interestingly, T. notatus can reduce the quality of N. attenuata as a host for Manduca spp. (Kessler and Baldwin 2004).

In summary, plant hormones and the metabolites they regulate are known to have a variety of effects on life history parameters of herbivores. The role of classical defense hormones such as JAs in herbivore performance has been intensively studied (Halitschke and Baldwin 2003; Steppuhn and Baldwin 2007; Wang et al. 2008). However, the role of classical growth hormones such as the CKs in mediating herbivore interactions is just beginning to be explored. Therefore, it would be interesting to study herbivores that use CKs to manipulate tissue development and sink strength in a similar way to the known roles of these hormones in mediating growth and development. It is known that T. notatus damage in $\mathrm{N}$. attenuata increased on tissue with elevated CK levels (Schäfer et al. 2013) and therefore, it is likely that increased levels of CKs benefit T. notatus. Manipulation of sex ratios has been shown to be an effective method in pest control (Hickey and Craig 1966; Galizi et al. 2014). From this perspective, it might be advantageous to alter the ability of $T$. notatus to manipulate CKs, yet this requires first revealing whether, and how, T. notatus manipulate CKs in their host plant.

\section{MATERIALS AND METHODS}

\section{Plant material and glasshouse conditions}

Seeds of an inbred line of the $31^{\text {st }}$ generation of Nicotiana attenuata Torr. Ex.Watts. (Solanaceae) were 
used as wild-type (WT) plants. The inbred line originated from seeds collected in 1988 from a natural population at the DI Ranch in southwestern Utah (Glawe et al. 2003). In addition to the WT plants, transgenic lines with reduced levels of jasmonic acid (JA;sLOX3; Halitschke and Baldwin 2003), decreased levels of 17-hydroxygeranyllinalool diterpene glycosides (DTGs, irGGPPS; Heiling et al. 2010), increased levels of CKs and protein levels at flowering (SAGipt4), silenced in two of the three predicted CK receptors (Schäfer et al. 2015) resulting in reduced defense induction and lower levels of primary metabolites (irCHK2/3), higher levels of trypsin protease inhibitor (TPI) and DTG accumulation but reduced levels of nicotine (irJAZh; Oh et al. 2012), or reduced photosynthetic rate and increased levels of RuBP protein (irRCA; Mitra \& Baldwin 2008; Mitra and Baldwin 2014) were used in glasshouse experiments. All lines used have already been published in the references cited, and the traits we aimed to manipulate have been demonstrated to be consistent in multiple independently transformed lines. The lines used have not shown evidence of off-target effects.

Seeds of WT and the transgenic lines were sterilized and germinated on Gamborg's B5 media as described by Krügel et al. (2002). After 10 days, seedlings were transferred to Teku pots. At $20 \mathrm{~d}$ postgermination, single plants were transferred to $1 \mathrm{~L}$ pots and grown at $26-28^{\circ} \mathrm{C}$ under $16 \mathrm{~h}$ of light as described by Krügel et al. (2002). Soil substrate consisted of Fruhstorfer-Nullerde mixed with $0.5 \mathrm{~g} / \mathrm{L}$ PG-Mix Compo 14-16-18, $0.0875 \mathrm{~g} / \mathrm{L}$ superphosphate, $0.35 \mathrm{~g} / \mathrm{L} \mathrm{MgSO}_{4} \times 7 \mathrm{H}_{2} \mathrm{O}$ and $0.05 \mathrm{~g} / \mathrm{L}$ Micromax. Plants were fertilized with Peters Allrounder $(20 \mathrm{~g} / 400 \mathrm{~L}$ day 7-14, $40 \mathrm{~g} / 400 \mathrm{~L}$ day $14-21,15-30 \mathrm{~g} / 400 \mathrm{~L}$ after $21 \mathrm{~d})$. Bor-Folicin was added to the substrate $(3 \mathrm{~g} / 400 \mathrm{~L}$ day $1-7,2 \mathrm{~g} / 400 \mathrm{~L}$ day $7-14,1 \mathrm{~g} / 400 \mathrm{~L}$ after $14 \mathrm{~d})$. After 6 weeks, plants were transferred to a remote glasshouse in Isserstedt, Germany, where we kept our insect colonies. Plants were then fertilized weekly with Peters Allrounder ( $1 \mathrm{~g} / \mathrm{L}$ water).

\section{Insect collection}

Empoasca sp. (Hemiptera: Cicadellidae) and Tupiocoris notatus Distant (Hemiptera: Miridae) were collected from Cucurbita foetidissima (Empoasca sp.) and $N$. attenuata plants (T. notatus) on and around Lytle Preserve in southwestern Utah and kept in the remote glasshouse of the Max Planck Institute for Chemical
Ecology in Isserstedt, Germany. The glasshouse colonies were already established as described in Kessler et al. (2004) and Kallenbach et al. (2012).

Number of T. notatus eggs, survival rate of parents, number of adult progeny and their sex ratio on $\mathrm{N}$.

\section{attenuata}

asLOX3 plants in the early elongation stage ( 6 weeks) were caged with freshly molted adults of $T$. notatus alone, or together with adult Empoasca sp. for 5-6 weeks $(n=7$ plants in the control condition and $n=8$ in the treatment with Empoasca sp., 10 [6 females +4 males of $T$. notatus] insects/species/plant). Freshly molted adult progeny of $T$. notatus were regularly collected, sorted into females and males and counted.

In order to estimate the number of eggs laid by T. notatus on N. attenuata plants, asLOX3 plants $(n=5)$ were infested either with $10 \mathrm{~T}$. notatus adults per plant (six females and four males) or simultaneously with Empoasca sp. adults (10/plant). After one week of oviposition, T. notatus were moved to new plants for another week and 10 new Empoasca sp. adults were added. This procedure was repeated three times. The entire plants were harvested and stained according to Backus et al. (1988) and the number of eggs was counted on each plant.

The survival of $T$. notatus adults (six females and four males per plant) was monitored on $\mathrm{N}$. attenuata plants (asLOX3) for 35 days when plants were, or were not co-infested with Empoasca sp. (10/plant, $n=9$ plants in the - Emp. and $n=10$ plants in the +Emp. treatment). The mortality of $T$. notatus adults was recorded and a Kaplan-Meier survival curve was constructed (Therneau 2015). To determine whether the sex ratio shift occurred due to mortality of progeny at the egg stage, ten $T$. notatus adults (six females and four males per plant) were reared on asLOX 3 N. attenuata plants and allowed to oviposit for one week. After oviposition, parents were removed and Empoasca sp. adults were added to roughly half of the plants (10/plant, $n=4$ plants in control treatment and $n=5$ plants in the treatment with Empoasca sp., Figure $4 \mathrm{~A}$ ) and the sex ratio of individuals reaching adulthood was determined. To determine whether differential mortality of nymphs could explain the sex ratio shift, $1^{\text {st }}$ instar nymphs of T. notatus were collected from the glasshouse colony and caged on asLOX3 plants either alone or with 
Empoasca sp. (10 insects/species/plant, $n=5$ plants, Figure 4D). The nymphs were monitored for 2 weeks until they reached adulthood and sorted into females and males.

To determine whether particular aspects of host plant quality play a role in the abundance or sex ratio of adult progeny, ten $T$. notatus adults per plant (six females + four males) were raised on early elongating $\operatorname{irGGPPS}(n=6), \operatorname{irCHK2} / 3(n=5)$ and control (WT, $n=6)$ plants and irJAzh and irRCA $(n=1$ and $n=2$, respectively). Similarly, $T$. notatus adults were monitored on flowering SAGipt4 $(n=6)$ and WT plants $(n=5)$, because the SAG promoter is not strongly activated until plants flower (Schäfer et al. 2015). Female and male progeny were also monitored on these plants. In addition, progeny were also counted on control (WT) and JA-deficient (asLOX3) plants $(n=10)$ to determine whether JA biosynthesis is involved in the sex ratio shift.

\section{Field observations}

Plants in two native populations of N. attenuata were surveyed once a week for a period of three weeks. Insects were counted and re-released on the same plants. The number of females and males was recorded $(n=16$ plants in population 1 and $n=10$ plants in population 2).

\section{PCR and pyrosequencing of the endosymbionts of $T$. notatus and Empoasca sp.}

$T$. notatus females were collected from two native populations ( $n=3$ for each population, N1- N6) and the glasshouse colony in Isserstedt after the insects fed on asLOX3 plants for one week ( $n=3 ; \mathrm{N7}-\mathrm{N} 9)$. All $T$. notatus samples that were labeled from $\mathrm{N} 1-\mathrm{N} 9$ and a pooled Empoasca sp. sample (five insects) from the glasshouse colony (N10) were screened for Wolbachia infection by PCR. DNA extraction was performed as described in Staudacher et al. (2016) and followed by PCR using general bacterial $16 \mathrm{~S}$ rRNA primers fD1 $_{1}$ (AGAGTTTGATCCTGGCTCAG) and rP2 (ACGGCTACCTTGTTACGACTT) as described in Weisburg et al. (1991). Wolbachia screening by PCR was performed using wsp81f (TGGTCCAATAAGTGATGAAGAAAC) and wsp691 (AAAAATTAAACGCTACTCCA) primers (Braig et al. 1998), and Drosophila melanogaster infected with Wolbachia pipientis was used as a positive control.
For pyrosequencing, DNA samples were sent to an external service provider (Research \& Testing Laboratories, Lubbock, USA) for bacterial tag-encoded FLX amplicon pyrosequencing (bTEFAP) with 16S rRNA primers Gray28F (5'-GAGTTTGATCNTGGCTCA-3') and Gray519R (5'-GTNTTACNGCGGCKGCTG -3') (Ishak et al. 2011; Sun et al. 2011). A sequencing library was generated through one-step PCR with 30 cycles, using a mixture of HotStar and HotStar HiFidelity Taq polymerases (Qiagen). Sequencing extended from Gray28F, using a Roche 454 FLX instrument with Titanium reagents and procedures at Research and Testing Laboratory (RTL, Lubbock, TX, USA, http:// www.medicalbiofilm.org/). Quality control and analysis of 454 reads was done in QIIME (Caporaso et al. 2010). Low-quality ends of the sequences were trimmed with a sliding window size of $50 \mathrm{bp}$ and an average quality cutoff of $25 \mathrm{bp}$. Subsequently, all low quality reads (quality cut-off $=25 \mathrm{bp}$ ), sequences $<200 \mathrm{bp}$, and putative chimeric reads (using usearch denovo as implemented in QIIME) were removed. High-quality reads were clustered into operational taxonomic units (OTUs) using a multiple-OTU-picking strategy with cdhit ( $\mathrm{Li}$ and Godzik 2006) and uclust (Edgar 2010), with 97\% similarity cut-offs. For each OTU, the most abundant sequence was chosen as the representative sequence. The RDP classifier was used for taxonomy assignment (Wang et al. 2007). An OTU table was generated describing the occurrence of bacterial phylotypes within the samples. Chloroplast OTUs were manually removed from the OTU table before generating rarefaction curves and bar plots.

These sequence data have been submitted to GenBank under BioProject accession number PRJNA338226.

\section{Statistical analyses}

Data were analyzed in R (R-Project; http://www.rproject.org). Binomial generalized linear models (GLM) were used to analyze the influence of Empoasca sp. co-infestation as well as the effect of the different transgenic lines on the sex ratio of $T$. notatus progeny. Poisson GLMs were applied to examine the impact of the co-infestation as well as the impact of the different transgenic lines on the total number of adult progeny, including female and male number. In cases of overdispersion, standard errors were corrected using quasi-GLM models. To test whether the survival of 
T. notatus adults differed between treatments, the data were analyzed with the Cox proportional hazard model (non-parametric survival model, Therneau 2015) and models were simplified by removing non-significant variables.

\section{ACKNOWLEDGMENTS}

We thank the glasshouse department at the Max Planck Institute for Chemical Ecology and the field team in 2015 for support. We also thank the technical staff at the department of Molecular Ecology for providing seeds of the transgenic lines as well as D. Veit from the workshop for his support of our glasshouse facility in Isserstedt. We thank N. van Dam for her feedback and we especially thank $M$. Schäfer for his comments and feedback on this manuscript. We also thank B. Weiss for his help with DNA extraction and we thank Brigham Young University for the use of their Lytle Preserve field station. We thank both the International Max Planck Research School (IMPRS) on the Exploration of Ecological Interactions with Chemical and Molecular Techniques and the Young Biodiversity Research Training Group - yDiv for their support of N.A.. This work was supported by the Max Planck Society (all); the German Centre for Integrative Biodiversity Research (iDiv) Halle-Jena-Leipzig, funded by the German Research Foundation (FZT 118; N.A., I.T.B., M.C.S.); European Research Council advanced grant Clockwork Green to I.T.B. (number 293926; I.T.B., M.C.S.), and in part by the Collaborative Research Centre Chemical Mediators in Complex Biosystems - ChemBioSys (CRC 1127) funded by the German Research Foundation (I.T. B., M.C.S.).

\section{AUTHOR CONTRIBUTIONS}

N. A. conceptualized and designed experiments, collected and analyzed the data, interpreted the data and wrote the manuscript. T. E. collected essential life history data. M. Kallenbach conceptualized and designed experiments, interpreted the data, and revised the manuscript. M. Kaltenpoth analyzed and interpreted pyrosequencing data and wrote the methods and results for these data. G.K. provided statistical advice and assisted with data analysis. I.T.B. conceptualized and designed experiments, interpreted the data, and revised the manuscript. M. C. S. conceptualized and designed experiments, interpreted the data, and revised the manuscript. All authors contributed to the finalization of the manuscript.

\section{REFERENCES}

Awmack CS, Leather SR (2002) Host plant quality and fecundity in herbivorous insects. Annu Rev Entomol 47: $817-844$

Backus EA, Hunter WB, Arne CN, Arne N (1988) Technique for staining leafhopper (Homoptera: Cicadellidae) salivary sheaths and eggs within unsectioned plant tissue. J Econ Entomol 81: 1819-1823

Lara MEB, Garcia MCG, Fatima T, Ehness R, Lee TK, Proels R, Tanner W, Roitsch T (2004) Extracellular invertase is an essential component of cytokinin-mediated delay of senescence. Plant Cell 16: 1276-1287

Birch MC, Svihra P, Paine TD, Miller JC (1980) Influence of chemically mediated behavior on host tree colonization by four cohabiting species of bark beetles. J Chem Ecol 6: 395-414

Braig HR, Zhou W, Dobson SL, O’Neill SL (1998) Cloning and characterization of a gene encoding the major surface protein of the bacterial endosymbiont Wolbachia pipientis. J Bacteriol 180: 2373-2378

Caporaso JG, Kuczynski J, Stombaugh J, Bittinger K, Bushman FD, Costello EK, Fierer N, Peña AG, Goodrich JK, Gordon JI, Huttley GA, Kelley ST, Knights D, Koenig JE, Ley RE, Lozupone CA, McDonald D, Muegge BD, Pirrung $M$, Reeder J, Sevinsky JR, Turnbaugh PJ, Walters WA, Widmann J, Yatsunenko T, Zaneveld J, Knight R (2010) QIIME allows analysis of highthroughput community sequencing data. Nat Methods 7: 335-336

Cédola CV, Ottaviano MFG, Brentassi ME, Cingolani MF, Greco NM (2013) Negative interaction between twospotted spider mites and aphids mediated by feeding damage and honeydew. Bull Entomol Res 103: 233-240

Charnov EL, Los-den Hartogh RL, Jones WT, van den Assem J (1981) Sex ratio evolution in a variable environment. Nature 289: $27-33$

Craig TP, Price PW, Itami JK (1992) Faculative sex ratio shifts by a herbivorous insect in responce to variation in host plant quality. Oecologia 92: 153-161

Denno RF, Peterson MA, Gratton C, Cheng J, Langellotto GA, Huberty AF, Finke DL (2000) Feeding-induced changes in plant quality mediate interspecific competition between sap-feeding herbivores. Ecology 81: 1814- 1827

Denno R, McClure M, Ott J (1995) Interspecific interactions in phytophagous insects: Competition reexamined and resurrected. Annu Rev Entomol 40: 297-331

Edgar RC (2010) Search and clustering orders of magnitude faster than BLAST. Bioinformatics 26: 2460-2461 
Faeth SH (1986) Indirect interactions between temporally separated herbivores mediated by the host plant. Ecology 67: 479-494

Fisher RA (1930) The genetical theory of natural selection. Oxford University Press, Oxford UK

Fox LR, Kester KM, Eisenbach J (1996) Direct and indirect responses of parasitoids to plants: Sex ratio, plant quality and herbivore diet breadth. Entomol Exp Appl 80: 289-292

Fox LR, Letourneau DK, Eisenbach J, Vannouhuys S (1990) Parasitism rates and sex ratios of a parasitoid wasp: Effects of herbivore and plant quality. Oecologia 83: 414-419

Fragoso V, Rothe E, Baldwin IT, Kim SG (2014) Root jasmonic acid synthesis and perception regulate folivore-induced shoot metabolites and increase Nicotiana attenuata resistance. New Phytol 202: 1335-1345

Galizi R, Doyle LA, Menichelli M, Bernardini F, Deredec A, Burt A, Stoddard BL, Windbichler N, Crisanti A (2014) A synthetic sex ratio distortion system for the control of the human malaria mosquito. Nat Commun 5: 3977

Glawe G, Zavala JA, Kessler A, Van Dam NM, Baldwin IT (2003) Ecological costs and benefits correlated with trypsin protease inhibitor productio in Nicotiana attenuata. Ecology 84: 79-90

Ha S, Vankova R, Yamaguchi-Shinozaki K, Shinozaki K, Tran LSP (2012) Cytokinins: Metabolism and function in plant adaptation to environmental stresses. Trends Plant Sci 17: 172-179

Halitschke R, Baldwin IT (2003) Antisense LOX expression increases herbivore performance by decreasing defense responses and inhibiting growth-related transcriptional reorganization in Nicotiana attenuata. Plant $\mathrm{J}$ 36: 794807

Halitschke R, Gase K, Hui D, Schmidt DD, Baldwin IT (2001). Molecular interactions between the specialist herbivore Manduca sexta (Lepidoptera, Sphingidae) and its natural host Nicotiana attenuata. VI. Microarray analysis reveals that most herbivore-specific transcriptional changes are mediated by fatty acid-amino acid conjugates. Plant Physiol 131:1894-1902

Hamilton WD (1967) Extraordinary sex ratios. A sex-ratio theory for sex linkage and inbreeding has new implications in cytogenetics and entomology. Science 156: 477-488

Heiling S, Schuman MC, Schoettner M, Mukerjee P, Berger B, Schneider B, Jassbi AR, Baldwin IT (2010) Jasmonate and ppHsystemin regulate key malonylation steps in the biosynthesis of 17-hydroxygeranyllinalool diterpene glycosides, an abundant and effective direct defense against herbivores in Nicotiana attenuata. Plant Cell 22: 273-292

Hickey WA, Craig GB (1966) Genetic distortion of sex ratio in a mosquito, Aedes aegypti. Genetics 53: 1177-1196

Inbar M, Eshel A, Wool D (1995) Interspecific competition among phloem-feeding insects mediated by induced hostplant sinks. Ecology 76: 1506-1515

Ishak HD, Plowes R, Sen R, Kellner K, Meyer E, Estrada DA, Dowd SE, Mueller UG (2011) Bacterial diversity in
Solenopsis invicta and Solenopsis geminata ant colonies characterized by $16 \mathrm{~S}$ amplicon 454 pyrosequencing. Microb Ecol 61: 821-831

Kallenbach M, Bonaventure G, Gilardoni PA, Wissgott A, Baldwin IT (2012) Empoasca leafhoppers attack wild tobacco plants in a jasmonate-dependent manner and identify jasmonate mutants in natural populations. Proc Nat Acad Sci USA 109: E1548-E1557

Kessler A, Baldwin IT (2001) Defensive function of herbivoreinduced plant volatile emissions in nature. Science 291: 2141-2144

Kessler A, Halitschke R, Baldwin IT (2004) Silencing the jasmonate cascade: Induced plant defenses and insect populations. Science 305: 665-668

Krügel T, Lim M, Gase K, Halitschke R, Baldwin IT (2002) Agrobacterium-mediated transformation of Nicotiana attenuata, a model ecological expression system. Chemoecology 12: 177-183

Li W, Godzik A (2006) Cd-hit: A fast program for clustering and comparing large sets of protein or nucleotide sequences. Bioinformatics 22: 1658-1659

Lynch ME, Kaplan I, Dively GP, Denno RF (2006) Host-plantmediated competition via induced resistance: Interactions between pest herbivores on potatoes. Ecol Appl 16: 855-864

Mathur V, Tytgat TOG, de Graaf RM, Kalia V, Sankara Reddy A, Vet LEM, van Dam NM (2013) Dealing with double trouble: Consequences of single and double herbivory in Brassica juncea. Chemoecology 23: 71-82

Mitra S, Baldwin IT (2008) Independently silencing two photosynthetic proteins in Nicotiana attenuata has different effects on herbivore resistance. Plant Physiol 148: $1128-1138$

Mitra S, Baldwin IT (2014) RuBPCase activase (RCA) mediates growth-defense trade-offs: Silencing $R C A$ redirects jasmonic acid (JA) flux from JA-isoleucine to methyl jasmonate (MeJA) to attenuate induced defense responses in Nicotiana attenuata. New Phytol 201: 13851395

Mousseau TA, Fox CW (1998) The adaptive significance of maternal effects. Trends Ecol Evol 13: 403-407

Myers JH (1978) Sex ratio adjustment under food stress: Maximization of quality or numbers of offspring? Am Nat 112: 381-388

Oh Y, Baldwin IT, Gális I (2012) NaJAZh regulates a subset of defense responses against herbivores and spontaneous leaf necrosis in Nicotiana attenuata plants. Plant Physiol 159: $769-788$

Robinson AS (1983) Sex-ratio manipulation in relation to insect pest control. Ann Rev Genet 17: 191-214

Schäfer M, Brütting C, Gase K, Reichelt M, Baldwin IT, Meldau S (2013) "Real time" genetic manipulation: A new tool for ecological field studies. Plant J 76: 506-518

Schäfer M, Meza-Canales ID, Navarro-Quezada A, Brütting C, Vanková R, Baldwin IT, Meldau S (2015) Cytokinin levels and signaling respond to wounding and the perception of 
herbivore elicitors in Nicotiana attenuata. J Integr Plant Biol 57: 198-212

Schuman MC, Heinzel N, Gaquerel E, Svatos A, Baldwin IT (2009) Polymorphism in jasmonate signaling partially accounts for the variety of volatiles produced by Nicotiana attenuata plants in a native population. New Phytol 183: $1134-1148$

Shine $R$ (1999) Why is sex determined by nest temperature in many reptiles? Trends Ecol Evol 14: 359-360

Siddique S, Radakovic ZS, De La Torre CM, Chronis D, Novák O, Ramireddy E, Holbein J, Matera C, Hütten M, Gutbrod P, Anjam MS, Rozanska E, Habash S, Elashry A, Sobczak M, Kakimoto T, Strnad M, Schmülling T, Mitchum MG, Grundler FMW (2015) A parasitic nematode releases cytokinin that controls cell division and orchestrates feeding site formation in host plants. Proc Nat Acad Sci USA 112: 12669-12674

Staudacher $\mathrm{H}$, Kaltenpoth $\mathrm{M}$, Breeuwer JAJ, Menken SBJ, Heckel DG, Groot AT (2016) Variability of bacterial communities in the moth Heliothis virescens indicates transient association with the host. PLoS ONE 11: e0154514

Stephens AEA, Srivastava DS, Myers JH (2013) Strength in numbers? Effects of multiple natural enemy species on plant performance. Proc R Soc Lond B Biol Sci 280: 20122756

Steppuhn A, Baldwin IT (2007) Resistance management in a native plant: Nicotine prevents herbivores from compensating for plant protease inhibitors. Ecol Lett 10: 499511

Strauss SY (1991) Direct, indirect, and cumulative effects of three native herbivores on a shared host plant. Ecology 72: 543-558

Sun Y, Wolcott RD, Dowd SE (2011) Tag-encoded FLX amplicon pyrosequencing for the elucidation of microbial and functional gene diversity in any environment. Methods Mol Biol 733: 129-141

Therneau $T$ (2015). A package for survival analysis in s. version 2.38, http://CRAN.R-project.org/package =survival

Thompson JN (1998) Coping with multiple enemies: 10 years of attack on Lomatium dissectum plants. Ecology 79: 2550-2554

Trivers RL, Willard DE (1973) Natural selection of parental ability to vary the sex ratio of offspring. Science 179: 90-92

Visser B, Le Lann C, Snaas H, Hardy ICW, Harvey JA (2014) Consequences of resource competition for sex allocation and discriminative behaviors in a hyperparasitoid wasp. Behav Ecol Sociobiol 68: 105-113

Wang L, Allmann S, Wu J, Baldwin IT (2008) Comparisons of LIPOXYGENASE3- and JASMONATE-RESISTANT4/6-Silenced plants reveal that jasmonic acid and jasmonic acid-amino acid conjugates play different roles in herbivore resistance of Nicotiana attenuata. Plant Physiol 146: 904-915

Wang Q, Garrity GM, Tiedje JM, Cole JR (2007) Naive Bayesian classifier for rapid assignment of rRNA sequences into the new bacterial taxonomy. Appl Environ Microbiol 73: 5261-5267

Weisburg WG, Barns SM, Pelletier DA, Lane DJ (1991) 16S ribosomal DNA amplification for phylogenetic study. J Bacteriol 173: 697-703

Werner T, Holst K, Pörs Y, Guivarc'h A, Mustroph A, Chriqui D, Grimm B, Schmülling T (2008) Cytokinin deficiency causes distinct changes of sink and source parameters in tobacco shoots and roots. J Exp Bot 59: 2659-72

Werner T, Schmülling T (2009) Cytokinin action in plant development. Curr Opin Plant Biol 12: 527-538

Werner T (1997) Biology of Wolbachia. Annu Rev Entomol 42: 587-609

Werren JH, Beukeboom LW (1998) Sex determination, sex ratios, and genetic conflict. Annu Rev Ecol Syst 29: 233261

West SA, Sheldon BC (2002) Constraints in the evolution of sex ratio adjustment. Science 295: 1685-1688

West, SA (2013) Sex ratios. In: Brenner's Encyclopedia of Genetics, 2nd edition, Vol. 6. Academic Press, San Diego. pp. 412-413

West SA, Reece SE, Sheldon BC (2002) Sex ratios. Heredity 88: 117-124

\section{SUPPORTING INFORMATION}

Additional supporting information may be found online in the supporting information tab for this article: http:// onlinelibrary.wiley.com/doi/10.1111/jipb.12507/suppinfo

Figure S1. The number of female and male of T. notatus adult progeny is not affected by Empoasca sp. coinfestation on asLOX3 plants

Graph show mean $\pm S D$. N. attenuata plants (asLOX 3 ) were infested either with $T$. notatus or simultaneously with Empoasca sp. and T. notatus. The number of female and male adult progeny of $T$. notatus did not differ between treatments. GLM; $n=8$ plants (in-Emp. treatment, one plant replicate died and thus, we excluded it from the experiment), 10 (six females + four males of $T$. notatus) insects/species/plant.

Figure S2. Survival curve of female and male T. notatus adults

Co- infestation with Empoasca sp. does not affect the survival rate of $T$. notatus adults on $\mathrm{N}$. attenuata plants. Graph shows a standard Kaplan-Meier-survival curve of $T$. notatus. The survival rate of $T$. notatus was monitored on $\mathrm{N}$. attenuata plants (asLOX 3 ) when plants were, or were not simultaneously infested with Empoasca sp. The survival of female and male parents did not differ between treatments. Cox proportional hazard models; 
$n=9$ plants in - Emp. treatment and $n=10$ plants in + Emp. treatment, 10 (six females + four males of $T$. notatus) insects/species/plant.

Figure S3. Agarose gel electrophoresis of PCR screen for Wolbachia infection in T. notatus

The red box indicates a sample from the field that is infected with Wolbachia. Glasshouse samples of $T$. notatus and Empoasca sp. were not infected. Drosophila melanogaster infected with $W$. pipientis was used as a positive control.

Figure S4. The number of T. notatus adult progeny and their sex ratio on wild-type (WT) and different transgenic lines of $N$. attenuata plants

Neither the sex ratio nor the number of female and male of T. notatus adult progeny differed on irGGPPS, irJAZh or irRCA plants versus WT. Graphs show counted male and female progeny as well as the sex ratio on each individual plant. (A) Control (WT), irGGPPS, irJAZh and irRCA plants of $N$. attenuata were infested with $T$. notatus. The number of female and male adult progeny of $T$. notatus and (B) the sex ratio did not differ between plants from the different genotypes. Since irJAZh and irRCA had low number of replicates $(n=1$ and $n=2$, respectively), statistical test was carried only for the comparison between control (WT) and irGGPPS plants. $\mathrm{GLM} ; n=6$ plants, 10 (six females + four males) insects/ plant.

Table S1. Final curated OTU table (abundances of all different OTUs across samples)

Table S2. OTU abundances across samples combined on the genus level

File S1. A representative sequence for each OTU in fasta format. OTU numbers correspond to those in the OTU table 